\title{
ALEXANDRE ET LA REINE DES AMAZONES
}

Michèle DAUMAS *

Résumé: - La plupart des historiographes d'Alexandre rapportent les amours du conquérant et de la reine des Amazones. Il s'agit en fait d'une fable moralisatrice destinée à mettre en garde
les Grecs contre les dangers de l'Orient.

Abstract. - Almost all the Alexander's chroniclers relate how the Amazones queen falled in protect the Greeks from the oriental dangers.

Peu de personnages mythologiques ont connu une aussi grande faveur auprès des artistes grecs que les Amazones. Simples guerrières s'affrontant aux Grecs sur les documents céramiques les plus anciens, on les confond vite, des l'apparition de la technique de la figure rouge, avec les Barbanes, Pen les croit établies. Plus tard, lorsque la Grèce a connu le choc des invasions perses, on les voit figurer sur nombre de monuments sculptés de Grèce ou d'Asie Mineure, combattant à pied ou à cheval, souvent blessées, souvent mourantes, mais toujours redoutables, symbole des dangers de la guerre barbare et du triomphe héroïque de leurs adversaires ${ }^{1}$ Les mythes racontent comment Héraclès, Achille, Thésée ont eu à les affronter les historiens d'Alexandre n'hésitent pas à franchir la frontière qui sépare l'histoire de la légende pour imaginer la rencontre du conquerrant et de leur reine. Incontestablement la présence de ces guerrières est constante dans l'imaginaire grec : elles fascinent les esprits

Étrangères à la civilisation grecque, les Amazones, venues d'un Orient lointain, le sont par leurs mœurs. Leur monde est exclusivement féminin et libéré. En dehors de la guerre, elles ne rencontrent les hommes que pour reproduire et se réservent le choix de leur partenaire. Alors que les femmes, dans le monde antique, ne sont destinées qu'à l'éducation de leurs enfants $\mathrm{e}$ aux tâches domestiques, elles, juchees sur leurs chevaux fougueux, guerrières accomplies. parcourent le monde, semant la terreur. Peut-être est-ce pour faire réflechir une jeune épouse à son bonheur dêtre femme en Grèce que sur un epinétron du Musée d'Athenes, inv. 907

* Université de Paris X-Nanterre.

作

$R E A, 94,1992$, nss $^{\text {os }}-4$, p. 347 à 354 


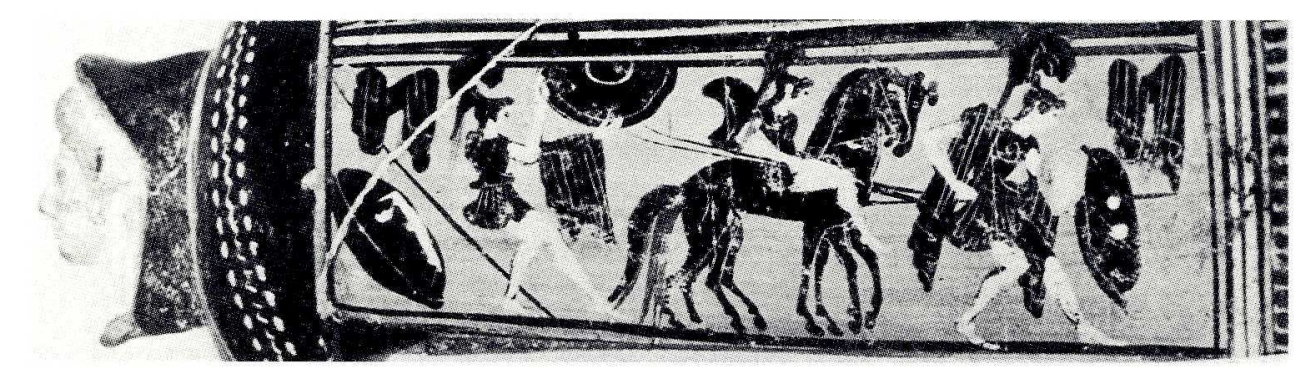

Fig. 1

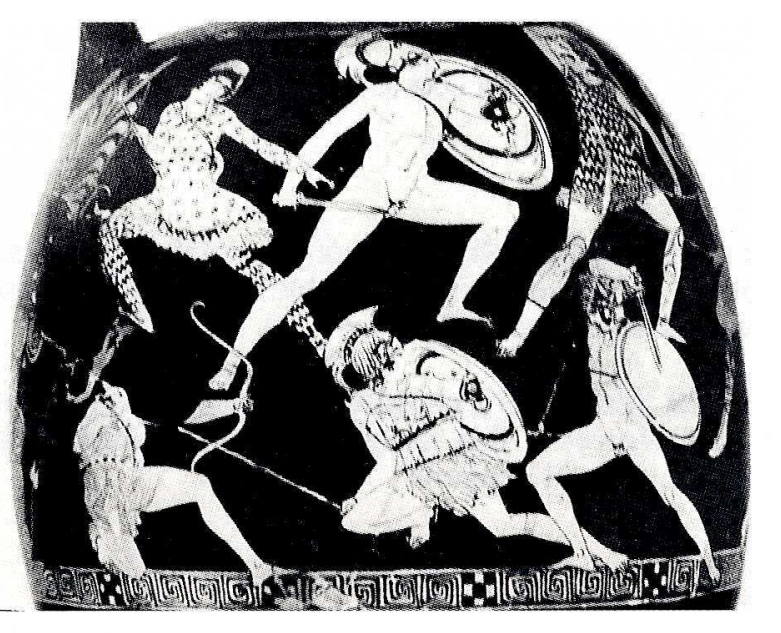

Fig. 3. - Berlin. Staatliche Museen.

Fig. 4. - Musée du Vatican. Fig. 5. - Musée du Capitole.
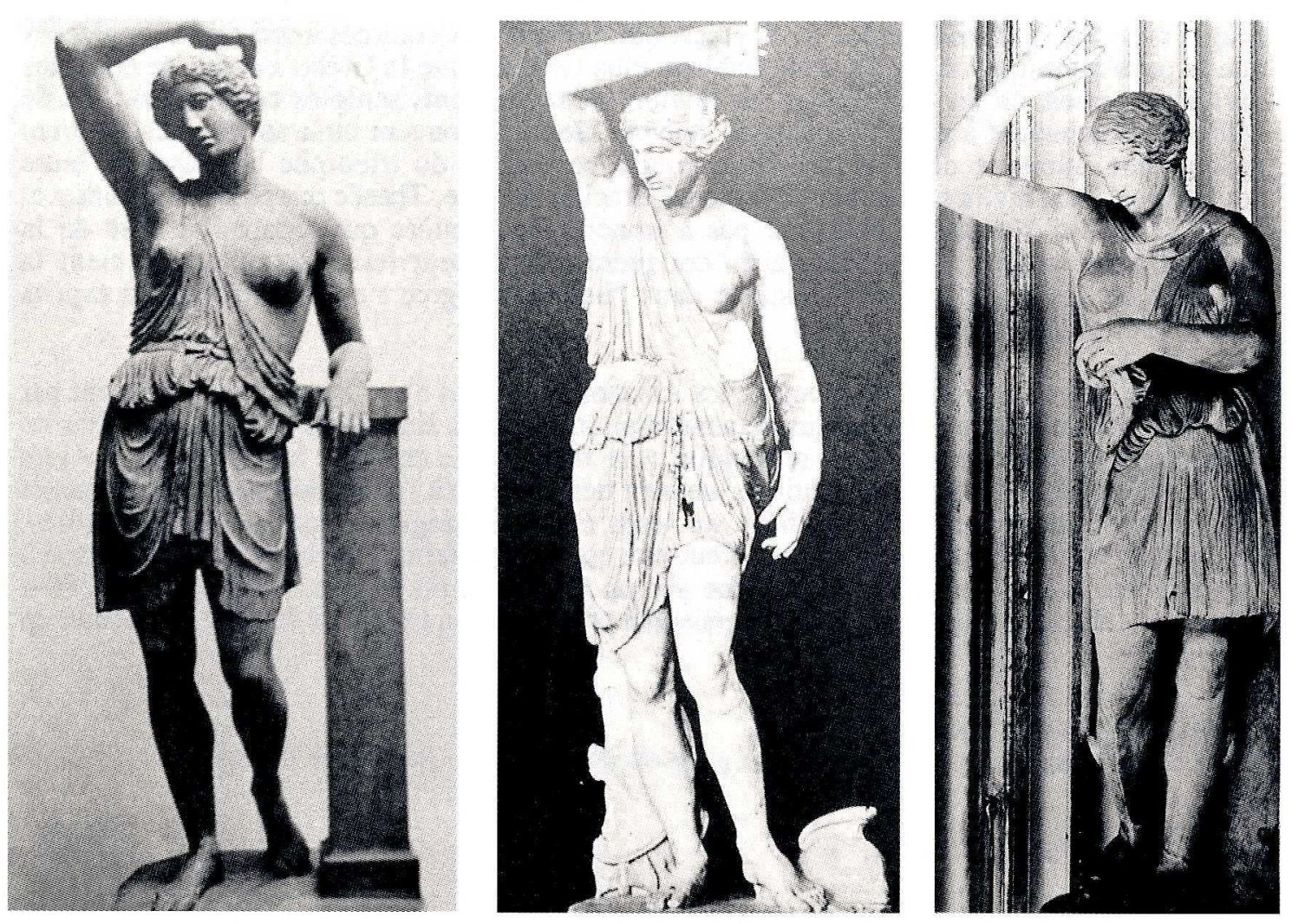
cadeau de mariage - l'artiste a représenté, au lieu des scènes traditionnelles de gynécée ${ }^{2}$, des Amazones fourbissant leurs armes avant le combat. La femme grecque est soumise, l'Amazone jeune Naxienne qui, dédiant une statue d'Artémis ${ }^{3}$ à Délos précise que « remarquable entre toutes, elle est fille de Deinodokos, sœur de Deinoménès, épouse de Pharos ». La femme grecque est une éternelle mineure, soumise à l'autorité des hommes de sa famille. Seule la alors que lamazi filles. Bien des femmes devaient secrètement envier une telle liberté

Les Amazones font également rêver les hommes et nourrissent leurs fantasmes. On les habille à la barbare, justaucorps bariolé dissimulant leurs formes féminines, ou on leur fai porter la tenue des guerriers grecs, tout en mettant en valeur leurs seins, comme sur le lécythe aryballisque de Naples, MNA, RC 239, où voisinent les deux types de représentation (Fig. 2) On raconte que pour mieux tirer de l'arc elles se brûlent le sein droit, mais on ne les représente jamais ainsi. Au contraire, au Ve siècle, les artistes qui concourent pour la statue de l'Amazone blessée dEphèse mettent tous en valeur la poitrine de la guenière, quils la repésentent les deux seins nus, comme Crés 4) (u Sosiclès (Fig 5) Ce dernier sculpteur compose la représentation la plus sensuelle : l'Amazone ayant détaché de son épaule gauche le chitoniskos dont elle est vêtue ${ }^{5}$ contemple la blessure qu'elle a reçue au flanc droit. La position du bras levé entraîne le gonflement du sein qui devient ainsi l'élément le plus important de l'image. Jamais

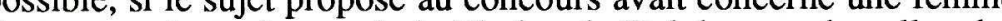
grecque. Et que dire des Amazones des metopes de la Tholos de Delphes ou de celles des .

La marque la plus flagrante de la barbarie des Amazones se situe dans l'organisation sociale de leur peuple, sorte de monde à l'envers qui bouleverse l'esprit rationnel des Grecs. Non seulement elles ont autrefois asservi les hommes au travail de la laine', mais leur activite principale est la guerre où elles excellent. Surtout, comme tous les peuples barbares, elles sont soumises à la monarchie. Sujettes d'une reine, elles ne peuvent être qu'esclaves aux yeux des reyaume est parfois situé en Cappadoce, au bord du Thermodon. Sa résidence royale se trouve dans la ville de Thémiscyra d'où elle s'élance à la tête de ses troupes pour des expéditions redoutables. Adversaire d'exception, elle s'affronte fatalement aux héros les plus prestigieux de a mythologie, quel que soit le nom qu'on lui donne selon les gênérations d'Amazones. Cest ainsi qu'Héraclès reçoit d'Eurysthée l'ordre de rapporter la ceinture d'Hippolyté, qu'Achille doi trop belle Antiope, l'e tent pas indemnes

2. Comme sur l'épinétron du peintre d'Frétrie, Athènes, Mus. Nat. inv. 1629, où sont représentées des scènes en rapport
(a) (1954) p. 29, fig. 4 sur un cpincto

3. Athènes Mus. Nat. inv. I.

4. Jadopte ici les attributions les plus couramment admises.

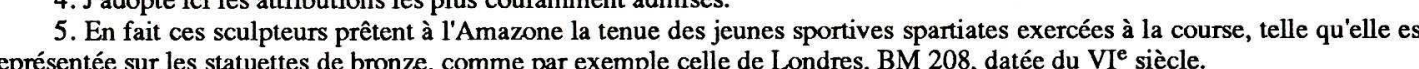

6. Pour la Tholos de Delphes, voir J. MARCADE BCH 110, (1986) p. 625-632 : pour le Mausolé, voir surtout les plaques

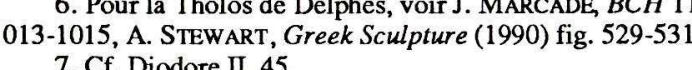


du combat : les guerrières détruisent leur équilibre psychologique. Héraclès est-il insensible à la beauté d'Hippolyte, lorsqu'il lui ravit sa ceinture, pièce de vêtement que le monde grec inspire à l'instant où il la tue Quant à Thésée, le rapt d'Antiope lui attirera bien des ennuis. Non seulement, jalouse de l'union qu'il contractera avec Phèdre, l'Amazone déclenchera la guerre, mais le fils qu'elle lui a donné, Hippolyte, périra d'une mort effroyable.

Nul doute, l'aventure des reines des Amazones et des héros grecs est bien d'ordre sexuel. La passion sulfureuse qu'elles inspirent n'entraine que désordre et destruction, déchaîne le Héraclès. Le héros se croyant victime de la trahison d'Hippolyte la tue. Achille en proie au coup de foudre que lui inspire Penthésilée mourante, tue Thersite qui le raille. Enfin Théséc doit faire face au peuple des Amazones assaillant Athènes et mettant en péril la ville nouvelle qu'il vient d'établir.

Femme attirante, irrésistible par le mystère de sa féminité masculine, l'Amazone conduit à était inévitable qu'Alexandre, devenu héros de légende, soit soumis, dans le mythe qui se créc autour de son personnage, à la fatalité d'une telle rencontre.

C'est en Hyrcanie que les auteurs anciens 8 qui le rapportent situent l'épisode. Darius mort, le conquérant s'élance à la poursuite de Bessos, lorsque vient le trouver la reine des Amazones.

Quinte-Curce se montre à ce sujet le plus disert des historiens d'Alexandre. Thalestris (tel est le Phase au Caucase, passe les frontières de son pays « brûlant du désir de voir Alexandre envoie d'abord une ambassade pour se faire annoncer et Alexandre ayant accepté sa visite, elle se présente à lui avec trois cents Amazones. Venue à cheval, elle saute à terre, ses deux lances en main, tunique courte dénudant la poitrine du côté gauche, c'est-à-dire sein découvert, puisque le sein droit est brûlé pour faciliter le tir à l'arc. (On croirait voir l'Amazone de Sosicles !). Thalestris est grande, Alexandre petit. La reine est très surprise car, precise QuinteCurce, «tous les barbares respectent une taille majestueuse ». Malgré cette déconvenue, elle Alexandre. Le conquérant sollicite d'elle une alliance guerrière : sans répondre, elle renouvelle sa requête. Vaincu par tant d'audace, Alexandre accepte et le couple se retire mais le souverain doit s'attarder treize jours à cette tâche d'un nouveau genre car «les désirs amoureux de la femme étaient plus ardents que les siens ». Puis chacun s'en retourne vers son destin : Thalestris regagne son royaume, Alexandre la Parthiène. L'histoire s'arrête là. Il n'est pas question d'une descendance.

Diodore de Sicile, l'auteur le plus ancien parmi ceux qui rapportent cette tradition, se montre plus sobre. A la diference de deux noms à la reine, Thalestris ou Minithya, reste également très bref. « Alexandre, dit-il, se donna treize jours de repos auprès d'elle . après quoi, jugeant qu'elle était grosse, elle sc retira $»^{10}$; il ne donne aucune précision sur l'enfant qui naquit de cette union.

8. Diodore de Sicile, XVII, 77, 1-3; Arrien VII, 13, 3; Quinte-Curce, VI, 5, 24-32; Justin, 12, 3; Plutarque, Vie d'Alexandre, 46, 1. Cf. E. MEDERE, Die Alexanderlegenden bei den ältesten Alexanderhistoriker, 1936, p. 84-93.

10. Traduction Didot, Aus, CuFs. 
Arrien et Plutarque ne cachent pas leur scepticisme, chacun à sa façon. Plutarque, confondant mythe et histoire, comme bien des Grecs, ne nie pas l'existence d'une reine des Amazones, mais la rencontre lui apparaît si improbable quili cite treize auteurs rapportant le Chambellan et Ptolémée « disent que c'est pure invention ». En fait, l'Amazone ne saurait être autre que la fille du roi des Scythes offerte en mariage au souverain. Et Plutarque de poursuivre : «du reste, que l'on ajoute foi ou non à ce récit, l'admiration pour Alexandre n'en sera ni diminuée ni accrue ».

Chez Arrien, Alexandre prend l'initiative de l'union et demande aux Amazones « d'annoncer à leur reine qu'il lui rendra visite pour lui faire un enfant $\gg^{12}$. L'historien se montre très principales. Il lui semble impossible qu'à l'époque d'Alexandre la race des Amazones ait encore existé, et même avant l'expédition du Macédonien, puisque Xénophon n'en dit rien. D'ailleurs, ose-t-il ajouter, « personnellement il me semble invraisemblable qu'une telle race de femmes ait tout simplement existé ». Alexandre a dû rencontrer « des femmes barbares exercées à l'équitation et ayant revêtu la tenue que la tradition prête aux Amazones ».

On croyait Alexandre descendant d'Héraclès et d'Achille ${ }^{13}$ : la rencontre avec l'Amazone ne pouvait qu'attester ces origines mythiques, aux yeux des nombreux auteurs qui exaltaient Alexandrie la Grande, rivale égytienne d'Athènes au déclin? Un tel récit grandissait encore la gloire du fils d'Ammon. Par ailleurs l'aventure était piquante et ne pouvait que charmer les lecteurs. C'est bien ainsi que Montaigne l'a lue. Il en fait un exemple pour illustrer son propos sur le désir amoureux ${ }^{14}$, episodique chez les hommes, toujours susceptible de naitre chez les femmes, quoique muet car "leur rolle est souffrir, obéir, consentir ». Seules les Amazones enfreignent la règle et osent déclarer leur passion, comme Thalestris trouvant Alexandre «s beau, jeune et vigoureux, elle qui estori parfaicte en toutes ses qualitez, lui conseilloit quils qui fust lors vivant, quelque chose de grand et de rare pour l'advenir ». L'auteur des $E$ ssais semble s'amuser beaucoup de l'aventure dont il ne retient que l'aspect romanesque et quelque peu scabreux, attitude qui devait être celle de la majorité des lecteurs des Vies d'Alexandre. Toutefois, les plus perspicaces comprenaient certainement que l'épisode prenait un tout autre sens, si on ne le détachait pas du contexte.

La rencontre du Macédonien et de la reine est une invention relativement tardive, puisque Arrien, aussi bien que Plutarque, affirment que ni Aristoboulos, hi Charès, hi Pive, pué n'e d'Alexandre les plus proches du pouvoir ${ }^{15}$. Le mythe a dû apparaître à la fin du IVe siècle

11. Sur les sources utilisées par Plutarque à à se sujet, cf. la notice de R. FLACELĖRE, Vie d'Alexandre, CUF, p. 11 à 23.
12. Traduction P. Savinel, Editions de Minuit. (1978)

13. Sur toutes ces questions, cf. Paul GouKowsKr, Essai sur les origines du mythe d'Alexandre I, Les origines politiques 14. Essais III, chapitre V (Pléiade, p. 991)

notamment été chargé de la restauration de la latiquuc, faisait partie de lexpédition en tant qu'architecte et ingénieur. II

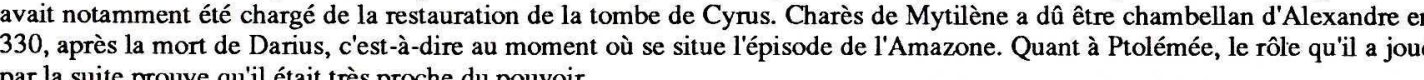


av. J.-C. ${ }^{16}$, à une époque où, tout en admirant le conquérant, on avait pris l'habitude de le juger D'alleurs, dès le vivant d'Alexandre on ne lui ménage pas les critiques sévères, comme en prétendue divinité mais on accepte très mal la politique de fusion des races entreprise après mort du Grand Roi. Reprenons les faits : la campagne d'Hyrcanie se situe en 330, au moment où Alexandre connaît les plus grandes difficultés. Il n'a pu prendre Darius vivant et Bessos entend bien assurer son pouvoir dans les satrapies orientales de la Drangiane, de la Sogdiane de la Bactriane. L'usurpateur n'a pu lui interdire l'entrée en Bactriane. Il le capture et le supplicie, mais Spitaménès suscite la guérilla en Sogdiane et en Bactriane. Il est temps pour Alexandre de prouver qu'il est le Grand Roi, le successeur de Darius. Lincendie de Persépolis a son châtiment. Il lui faut dés: : leurs coutumes : il exige la proskynèse, porte les vêtements royaux, robe rouge et blanche, diadème, s'entoure d'un harem ${ }^{18}$, sert des festins aux dignitaires ${ }^{19}$. Les conséquences de cette conduite seront terribles : d'abord le meurtre de Cleitos qu'Alexandre tue au cours d'une beuverie, ce compagnon darmes lui ayant reproché dans son ivresse de se faire prendre pour un dieu et de se vêtir comme un Barbare ${ }^{20}$. Puis ce fut l'affaire de la proskynèse que les Macédoniens refusèrent d accepter ${ }^{21}$, la concédant aux seuls Orientaux. Enfin, il eut à faire face que le souverain fit exécuter, laqume s'il voulait faire taire ce mâtre lointain qui correspondance le prouve22 - voulait que son disciple cherchât sa gloire ailleurs que dans faste d'une cour barbare.

En fait, aux yeux des Grecs et des Macédoniens la faute d'Alexandre était de devenir barbare, au lieu de soumettre les Perses à lhellenisme. Quinte-Curce, VI, 6, 10, termine son long exposé sur la corruption royale qui suit l'episode de l'Amazone par ces réflexions des vaincus qu'aux vainqueurs, s'était mué, de chef de la Macédoine en satrape de Darius e aux concessions faites à l'Orient ne pouvaient qu'être pernicieuses pour l'Occident $:$ l'intelligence grecque se diluerait à coup sûr dans la mollesse de telles mours. Tel est le sens du récit des

16. Puisque, aux dires de Plutarque, Cleitarchos, Polycheitos, Onésicritos qui avaient participé à l'expédition ne niaient
l'épisode. Le mythe a dûn nâtre très vite après la mort d'Alexandre et connâtre aussitôt la célébrité en rai son du caractère fantastique d'une telle rencontre qui renforçait l'aura surnaturelle du conquérant.

年 honneurs divins au roi dans les cités grecques. L'affaire du culte d'Alexandre, portée à Athènes devant l'assemblée, entraîn l'adhésion passionnée de Démade, mitigée de Démosthene, et la farouche opposition de Lycurgue et Hypéride. Démade fu 18. En fait il récupéra le harem de Darius qui avait

19. Les festins servis par Alexandre aux Macédoniens et aux Perses furent nombreux et somptueux, les plus célebres
s. 20. Sur le meurtre de Cleitos, cf. P. GoukowsKY, o. .c. p. 44-47.

21. Le refus des Grecs venait de la confusion qu'ils établissaient entre le salut fait par ses sujets au Grand Roi, tel qu'on le voit représenté sur les reliefs de Persépolis, et le geste dadoration reservé aux dioux. Sur toute cette affaire,
cf.P. GouKowsKY, o.c.,p. $47-49$.

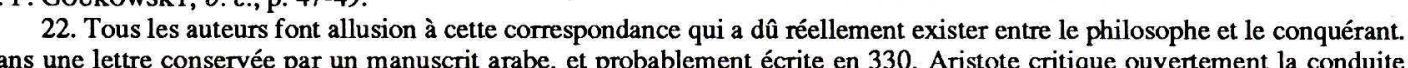
Dans une lettre conservée par un manuscrit arabe, et probablement écrite en 330 , Aristote critique ouvertement la conduite
d'Alexandre et le rappelle à ses devoirs : imposer l'homonoia et la philia en Grèce en créant une cité unique, être roi des Grecs et des Perses en privilégiant les premiers aux dépens des seconds quil faut déporter en Europe et en Grèce ; il ne doit pas devenir un tyran comme les rois barbares, mais au contraire légifiérer et fonder des cités. Authentique ou non, cette lettre
témoigne de l'influence qu'Aristote entendait exercer sur son disciple et dont il ne devait pas se cacher. Sur toutes ces

témoigne de l'influence qu'Aristote entendait exercer sur son disciple et dont il ne devait pas se cacher. Sur toutes ces 
amours d'Alexandre et de Thalestris, qui se présente, chez tous les auteurs qui y croient, comme une fable moralisatrice. C'est après ces treize jours passés dans les bras de l'Amazone est d'ordre moral - des vêtements barbares, donc effeminés, un harem considérable, des beuveries et leurs conséquences allant jusqu'au meurtre des amis - et d'ordre politique agitations et révoltes, conspirations et exécutions. La morale de la fable est partout clairement dégagée. « Après quoi ( $\mu \varepsilon \tau \dot{\alpha} \delta \hat{\varepsilon} \tau \alpha \hat{v} \tau \alpha)$, dit Diodore (XVII, 4), s'imaginant qu'il avait désormais réussi son entreprise et que nul ne lui disputerait plus la possession de l'Empire, il se mit rechercher passionnement le luxe perse et la magnificence des rois asiatiques ». Quinte-Curce $(V I, 6,1)$ voit dans les mesures prises après la rencontre le déchaînement des passions du Justin (12,3), il relie encore plus brutalement l'épisode de l'Amazone et l'orientalisetion du souverain : "ce fut alors (post haec) qu'Alexandre adopta le costume des rois de Perse."

Arrien, nous l'avons vu, ne croit pas au mythe des Amazones et pourtant il ne peut s'empêcher d'évoquer les preuves tangibles de leur existence que sont à ses yeux la tradition littéraire et les ouvres d'art ${ }^{23}$. En fait, lui qui admire parfois sans la plus élémentaire réserve
Alexandre ne peut admettre que son héros soit coupable d'une si lourde erreur. Et c'est egalement lopinion de Plutarque, le moraliste. Même si l'histoire était véridique, en quoi cela immense rachète largement les faiblesses orientaes.

Soumettez l'Orient, ne vous laissez pas aller à en adopter les coutumes, telle est la morale de la fable. Thalestris, comme ses sœurs les Amazones, est en quelque sorte l'allégorie de la Barbarie qui règne sur ces vastes territoires où les Grecs ont toujours rêvé de s'aventurer. Les récits de la guerre de Troie n'en sont-ils pas le témoignage 24 ? Mieux que tout autre image, l'accoutrement de i'Amazone, son corps superbe de sportive, sa liberté de mours pouvaien d'une faune et d'une flore exotiques, exerçait sur les Grecs. Bijoux et vaisselle d'or, peuplés tentures chatoyantes, faste d'une cour qui se déplaçait tout entière avec le Roi, lorsqu'il faisait la guerre, devaient faire rêver plus d'un modeste citoyen 25 . La fable rappelle que le génie de la Grèce est ailleurs que dans le luxe et la mollesse. Comme disait Hérodote, « la Grèce a toujours eu la pauvreté pour sour de lait », mais l'hellénisme, pour conquérir le monde, n'a pas besoin de la richesse orientale. Seule la vertu de ses citoyens a fait sa grandeur. Lepoque hellenistique, durant laquelle s'est très probablement creée la fable d'Alexandre et de Thalestris, est justement celle où les antiques valeurs de la cité se sont dissoutes au profit du pas inutile de faire réfléchir les admirateurs d'Alexande aux dangers de la vie facile que leur

apportait la domination de l'Orient.

23. VII, 13, 5-6 : « ce combat entre les Athéniens et les Amazones fut peint par Cimon dans un tableau d'aussi grande

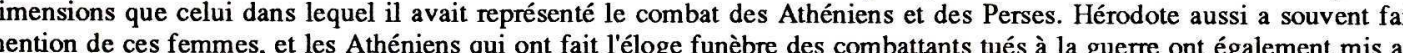
premier plan cette action des Athéniens contre les Amazones.»
24. La lecture de I'lliade est revélatrice à cet égard. Troie est une ville splendide, la cour de Priam fastueuse, la plaine 25. II suffit de relire pors

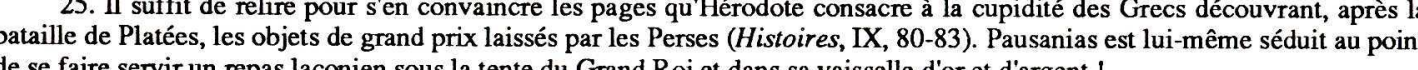


Ainsi, même à une époque tardive, les Amazones sont bien vivantes dans l'imaginaire grec, pour l'homme du peuple qui contemple leur image partout présente sur les monuments de sa ville comme pour l'intellectuel qui a beaucoup de mal à ne pas croire à leur existence. Bien conquérant et de la reine des Amazones est révélateur de toute une mentalité. L'attrait du Barbare n'est pas moins grand que la crainte qu'il inspire et la supériorité grecque, face à lui, se situe dans une marge très étroite où le héros rique la perdition morale. Héraclès, Achille, Thésée, Alexandre, victimes de cette séduction, vont bravement au devant du danger : leur courage les perd. Femmes travesties, féminines et masculines à la fois, symbole de l'exotisme et de la transgression, les Amazones n'ont pas fini de faire rêver. Les écuyères de nos cirques, chapeau haut-de-forme et redingote etroitement ceintree, pantalons et bottes de cheval, et dominatrices? En ayant recours au mythe des Amazones, les historiens d'Alexandre étaient assurés de frapper les esprits : Le Macédonien avait été aimé de leur reine, c'était sa gloire : Thalestris avait réussi à faire de lui un Oriental, c'était la faiblesse du conquérant, le modèle à ne pas suivre. 